\title{
Experimental optimization of composite collapsible tubular energy absorber device
}

\begin{abstract}
A four-phase program to improve the specific energy absorbed by axially crushed composite collapsible tubular energy absorber devices was undertaken. In the first phase, examining of the crushing behaviour of non-triggered tubes was carried out. The second phase is aimed at obtaining the best position for the triggered wall. The third phase focuses on the effects of material sizing in order to understand the influence of triggered wall length on the responses of composite circular tubes to the axial crushing load. The results of these three phases of the study contribute to the fourth whose objective is to optimize the shape geometry of the crosssection area to further improving in tube energy absorption capability. The experimental results demonstrated the strong potential benefits of optimizing the material distribution. The sizing and shape optimization of composite collapsible tubes exhibited a pronounced effect on their capability to absorb high specific energy under axial compressive load.
\end{abstract}

Keyword: Optimization, Sizing, Shape, Composite, Energy absorber, Tube 